\title{
Comparaison de divers modes d'agitation des échantillons de lait cru en vue de la numération de sa flore totale suivant la méthode Thompson
}

\author{
par \\ J. RICHARD*, C. KREBS**, J. WALTER $+* * *$ \\ et R. GRAPPIN****
}

\section{INTRODUCTION}

La méthode simplifiée de numération de la flore microbienne décrite par Thompson et al. [14] est largement utilisée en France pour le paiement du lait cru suivant sa qualité bactériologique. A condition d'être utilisée correctement, elle donne, avec les laits réfrigérés, des résultats comparables à la méthode classique par dilutions $[6,8,9,10,11,13,14]$. Cependant certains auteurs et plus récemment Grappin [3] ont constaté qu'elle conduit parfois à des résultats sous-estimés par rapport à ceux de la méthode classique surtout lorsque les échantillons contiennent plus de 100000 microorganismes/ml $[13,16]$.

Pour Wright et al. [16] cette sous-estimation serait due au "surpeuplement " des boîtes de Petri. Tatini et al. [13], observant que l'écart entre les deux méthodes de numération dépend aussi de l'origine des échantillons, ont émis l'hypothèse que la nature de la flore joue un rôle important : le lait de bidons contiendrait en majorité des microcoques et des streptocoques formant des amas et des chaînes et le lait de tanks, des bâtonnets isolés ou en petits paquets. L'agitation des tubes de dilution, en numération classique, serait responsable d'un fractionnement des amas microbiens plus important qu'en

* I.N.R.A., Laboratoire de Microbiologie Laitière et de Génie Alimentaire 78350 Jouy-en-Josas.

** Laboratoire de l'I.C.A.L.Y.N. - 89470 Moneteau.

*** Faculté de Pharmacie de Strasbourg, Laboratoire d'Analyses et de Recherches Bromatologiques et Nutritionnelles - 67000 Strasbourg.

**** I.N.R.A., Station Laitière - 39800 Poligny. 
numération simplifiée [4]. Cela expliquerait que l'écart entre les deux méthodes de dénombrement n'apparaît important que dans des cas particuliers.

Bien que cette explication ne soit pas entièrement satisfaisante (elle ne s'applique pas au lait réfrigéré qui contient une majorité de bâtonnets) et que l'écart entre les deux techniques de numération peut parfois être dû aussi à un défaut de calibrage de la boucle ou à sa mauvaise utilisation $[1,3]$, il est certain que le mode d'agitation du lait peut influencer les résultats des dénombrements [7, 11]. Il est donc important de le définir. Le présent article regroupe les résultats d'une étude de divers modes d'agitation du lait cru entreprise dans ce but.

\section{METHODES}

\section{Préparation des échantillons}

Des échantillons ont été prélevés dans différentes fermes juste après la traite ou après conservation du lait à basse température en tanks réfrigérants. Chaque échantillon, d'un volume de 0,5 1 environ, a été agité au laboratoire, suivant le mode AS défini plus loin, puis réparti en flacons de type pilulier à large ouverture, de sorte que ces derniers soient au plus à moitié pleins. Après $24 \mathrm{~h}$ de conservation entre $0^{\circ} \mathrm{C}$ et $5^{\circ} \mathrm{C}$, chaque flacon était agité suivant l'un des modes ci-dessous.

\section{Modes d'agitation}

Une revue de la littérature et une enquête réalisée auprès de divers laboratoires nous ont incité à retenir, pour comparaison, les huit modes d'agitation suivants :

S5 et S25
S25RO, S25R10 et S25R20

respectivement 5 et 25 simples retournements du flacon.

réchauffement du lait à $30-37^{\circ} \mathrm{C}$ en $5 \mathrm{~min}$, suivi de l'agitation S25, l'analyse étant faite sans délai (S25RO) après une attente de $10 \mathrm{~min}$ (S25R10) ou de $20 \mathrm{~min}$ (S25R20).

AS agitation "standard " [2] consistant en vingt-cinq mouvements aller et retour d'une amplitude de $25-30 \mathrm{~cm}$ effectués en 7 à $10 \mathrm{~s}$.

V agitation mécanique à l'aide d'un vibrateur de type Vortex réglé au maximum.

UT

traitement pendant $30 \mathrm{~s}$ à l'aide d'un homogénéiseur à turbine (Ultraturrax, type TP $18 / 2 \mathrm{~K}$ ) tournant à $20000 \mathrm{t} / \mathrm{min}$. 
Immédiatement après ce traitement, on effectuait un transvasement du lait pour éliminer l'épaisse couche de mousse stable qui se forme et qui empêche de faire un prélèvement correct avec l'anse calibrée.

L'analyse était faite dans la minute qui suit l'agitation sauf dans les cas S25R10 et S25R20 où elle était différée respectivement de 10 et de $20 \mathrm{~min}$.

\section{Méthodes d'ensemencement des boîtes de Petri}

Trois laboratoires ont participé à cette étude. Dans deux d'entre eux le prélèvement avec l'anse calibrée était réalisé manuellement, en veillant bien à ce que la vitesse de retrait soit toujours la même et que la boucle demeure dans un plan vertical au moment de sa sortie du lait. Dans le troisième, cette opération était mécanisée au moyen d'un dispositif à crémaillère entraîné par un moteur électrique, afin que la vitesse et l'angle de la boucle demeurent aussi constants que possible [11].

L'eau de rinçage présente dans la boucle était éliminée soit à l'aide d'un papier filtre, soit par un petit coup sec appliqué sur la canule portant la boucle.

Dans une première étude (ne comprenant pas le mode d'agitation UT) nous avons évité de plonger l'anse dans la couche de mousse ; pour cela on inclinait le flacon (méthode manuelle), ce qui avait pour effet de dégager une surface apparemment exempte de mousse.

Une deuxième étude, limitée aux modes d'agitation S25, AS et UT a été réalisée pour étudier l'influence de la couche superficielle du lait, et en particulier de la mousse, sur les résultats des dénombrements. Dans ce cas, les prélèvements avec l'anse étaient réalisés dans la minute qui suivait l'agitation, puis $10 \mathrm{~min}$ plus tard, avant et après élimination, par transvasement, de la couche superficielle, le lait étant conservé au froid entre la première et la deuxième analyse.

Le nombre d'échantillons utilisés par chaque laboratoire dans chacune de ces deux expériences est indiqué dans les tableaux 1 et 4 .

Les dénombrements ont été faits en double et dans un cas en triple, sur milieu gélosé Plate Count Agar (Difco $\left.n^{\circ} 429\right)$, les boîtes de Petri étant incubées 3 j à $30^{\circ} \mathrm{C}$.

\section{Exploitation des résultats}

L'ensemble des résultats des dénombrements microbiens a été soumis à une analyse de variance après transformation logarithmique des données. Nous avons pris le mode d'agitation AS (agitation "standard ") comme référence et nous avons comparé les moyennes en prenant la plus petite différence significative comme critère [12]. 


\section{RESULTATS ET DISCUSSION}

Les résultats moyens des dénombrements effectués au cours de la première étude sont présentés dans le tableau 1 et les résultats de l'analyse statistique, dans les tableaux 2 et 3 . Les laboratoires A et B trouvent des différences significatives entre les divers modes d'agitation alors que le laboratoire $\mathrm{C}$ n'en trouve pas. Comme on pouvait s'y attendre avec S5, S25 et S25R0 (échantillons faiblement agités) le nombre moyen de colonies par boîte de Petri est généralement inférieur à celui que l'on obtient avec la méthode AS prise comme référence. Il en est de même, mais dans une moindre mesure, de l'agitation par le Vortex. Par contre, on obtient des valeurs généralement supérieures à celles données par la référence AS lorsque les échantillons réchauffés à $37^{\circ} \mathrm{C}$ attendent $20 \mathrm{~min}$ avant d'être examinés (mode S25R20) alors que l'agitation est la même qu'avec S25R0.

Le tableau 2 montre que dans les laboratoires A et B, on observe une interaction hautement significative $(P<0,001)$ entre modes d'agitation et échantillons. Cela signifie que l'efficacité de l'agitation varie suivant l'origine des échantillons, donc probablement suivant la nature de la flore microbienne du lait, et/ou suivant la nature même de ce dernier [11]. On notera que la "résiduelle » (variance de répétabilité) est globalement un peu supérieure à la limite admise de 0,0050 pour la méthode classique de dénombrement [2].

Dans le tableau 3, on présente l'erreur "résiduelle " correspondant à chacun des modes d'agitation. On n'observe pas de différence importante de répétabilité entre ces derniers, ni entre prélèvement manuel (laboratoires A et B) et prélèvement mécanisé avec l'anse calibrée (laboratoire C). Les valeurs observées dépassent presque toutes la limite de 0,0050 .

Les résultats de la deuxième étude, portant sur l'influence de la couche superficielle du lait, sont présentés dans le tableau 4. D’une façon générale, on observe que la couche superficielle du lait, après $10 \mathrm{~min}$ de repos, contient beaucoup plus de micro-organismes (environ 50 p. 100 en plus avec S25 et UT) que le lait privé de cette couche ; par contre, dans ce dernier, le nombre de micro-organismes n'est pas significativement différent de celui qu'on obtient en faisant le prélèvement à l'anse, dans la minute qui suit l'agitation. Comme dans la précédente expérience S25 donne des résultats en moyenne inférieurs à ceux obtenus avec AS ; par contre, l'agitation avec l'Ultraturrax entraîne une augmentation du nombre de colonies de 40 à 100 p. 100 par rapport à cette référence, ce qui est bien en accord avec les résultats de Wanser et Hartman [15] et de Richard [11].

Sur le plan de la répétabilité des dénombrements, on n'observe pas de différence marquée entre les trois modes d'agitation comparés. Cependant, cette répétabilité est, dans l'ensemble, meilleure dans 
TABLEAU 1

Comparaison des divers modes d'agitation du lait

(Moyenne logarithmique du nombre de colonies microbiennes par boîte de Petri)

\begin{tabular}{|c|c|c|c|c|c|c|c|c|}
\hline \multirow{2}{*}{ Laboratoire } & \multirow{2}{*}{$\begin{array}{c}\text { Nombre } \\
\text { d'échantillons }\end{array}$} & \multicolumn{7}{|c|}{ Mode d'agitation } \\
\hline & & $\mathrm{S}_{5}$ & $\mathrm{~S}_{25}$ & $\mathrm{~S}_{25} \mathrm{R}_{0}$ & $\mathrm{~S}_{25} \mathrm{R}_{10}$ & $\mathrm{~S}_{25} \mathrm{R}_{20}$ & AS (c) & $\mathrm{V}$ \\
\hline A (1) & $\begin{array}{r}7 \text { (a) } \\
27 \text { (a) }\end{array}$ & $1 \overline{10^{* *}}$ & $\begin{array}{l}600^{* *} \\
117^{* *}\end{array}$ & $\begin{array}{l}710^{* *} \\
123\end{array}$ & $\begin{array}{l}1070 \\
141^{*}\end{array}$ & $\begin{array}{c}2500^{* *} \\
141^{*}\end{array}$ & $\begin{array}{r}1050 \\
129\end{array}$ & $\begin{array}{l}890^{* *} \\
120^{*}\end{array}$ \\
\hline B (1) & 19 (a) & $60^{* *}$ & $60^{* * *}$ & $62^{* *}$ & 80 & $115^{* *}$ & 81 & - \\
\hline C (2) & 6 (b) & - & 93 & 87 & 91 & 87 & 89 & 95 \\
\hline
\end{tabular}

(1) et (2) Prélèvement respectivement manuel et mécanique avec l'anse calibrée.

(a) Numération en double.

(b) Numération en triple.

(c) Méthode prise comme référence.

* et ** Moyenne significativement différente de celle donnée par le mode d'agitation AS aux seuils $\mathrm{P}=0,05$ et $\mathrm{P}=0,01$ respectivement. 
TABLEAU 2

Analyse de variance des résultats du tableau 1

\begin{tabular}{|c|c|c|c|c|}
\hline $\begin{array}{c}\text { Origine } \\
\text { des variations }\end{array}$ & Laboratoire & $\begin{array}{l}\text { Degré } \\
\text { de liberté }\end{array}$ & Carré moyen & $\mathrm{F}$ \\
\hline Entre échantillons & $\begin{array}{l}\text { A } \\
\text { B } \\
\text { C }\end{array}$ & $\begin{array}{r}26 \\
18 \\
5\end{array}$ & $\begin{array}{l}2,127 \\
1,855 \\
4,530\end{array}$ & $\begin{array}{l}348,7^{*} \\
187,4^{*} \\
573,4^{*}\end{array}$ \\
\hline Entre modes d'agitation & $\begin{array}{l}\mathrm{A} \\
\mathrm{B} \\
\mathrm{C}\end{array}$ & $\begin{array}{l}6 \\
5 \\
5\end{array}$ & $\begin{array}{l}0,0887 \\
0,4619 \\
0,0042\end{array}$ & $\begin{array}{l}14,54^{*} \\
46,66^{*} \\
<1,00\end{array}$ \\
\hline 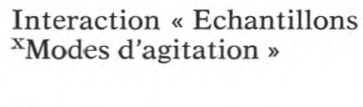 & $\begin{array}{l}\mathrm{A} \\
\mathrm{B} \\
\mathrm{C}\end{array}$ & $\begin{array}{r}156 \\
90 \\
26\end{array}$ & $\begin{array}{l}0,0135 \\
0,0328 \\
0,0126\end{array}$ & $\begin{array}{l}2,21^{*} \\
3,31^{*} \\
1,59\end{array}$ \\
\hline $\begin{array}{l}\text { Résiduelle } \\
\text { (variance de répétabilité) }\end{array}$ & $\begin{array}{l}\mathrm{A} \\
\mathrm{B} \\
\mathrm{C}\end{array}$ & $\begin{array}{r}189 \\
114 \\
72\end{array}$ & $\begin{array}{l}0,0061 \\
0,0099 \\
0,0079\end{array}$ & \\
\hline
\end{tabular}

* $\mathrm{P}<0,01$.

\section{TABLEAU 3}

Influence du mode d'agitation du lait sur la variance de répétabilité (erreur résiduelle) de la méthode simplifiée de numération (Variance x $10^{4}$ sur $\log _{10}$ )

\begin{tabular}{|c|c|c|c|c|c|c|c|c|}
\hline \multirow{2}{*}{ Laboratoire } & \multirow{2}{*}{$\begin{array}{c}\text { Degré de } \\
\text { liberté }\end{array}$} & \multicolumn{7}{|c|}{ Méthode d'agitation } \\
\hline & & $\mathrm{S}_{5}$ & $\mathrm{~S}_{25}$ & $\mathrm{~S}_{25} \mathrm{R}_{0}$ & $\mathrm{~S}_{25} \mathrm{R}_{10}$ & $\mathrm{~S}_{25} \mathrm{R}_{20}$ & AS & V \\
\hline A & 27 & 87 & 62 & 62 & 59 & 49 & 46 & 65 \\
\hline B & 19 & 64 & 68 & 171 & 58 & 160 & 74 & - \\
\hline $\mathrm{C}$ & 12 & - & 41 & 146 & 34 & 80 & 80 & 93 \\
\hline Moyenne & & 78 & 60 & 116 & 54 & 92 & 63 & 74 \\
\hline
\end{tabular}


Influence de la couche superficielle du lait sur le dénombrement de la flore microbienne suivant la méthode Thompson (Moyenne logarithmique du nombre de colonies par boîte de Petri)

\begin{tabular}{|c|c|c|c|c|c|c|c|c|c|c|}
\hline \multirow{3}{*}{ Laboratoire } & \multirow{3}{*}{$\begin{array}{c}\text { Nombre } \\
\text { d'échantillons }\end{array}$} & \multicolumn{9}{|c|}{ Mode d'agitation } \\
\hline & & \multicolumn{3}{|c|}{$\mathrm{S}_{25}$} & \multicolumn{3}{|c|}{ AS } & \multicolumn{3}{|c|}{ UT } \\
\hline & & a & $\mathrm{b}$ & c & a & $\mathrm{b}$ & c & a & $\mathrm{b}$ & c \\
\hline A & 11 & $\begin{array}{c}75 \\
(0,0055)\end{array}$ & $\begin{array}{c}96 \\
(0,0071)\end{array}$ & $\begin{array}{c}84 \\
(0,0029)\end{array}$ & $\begin{array}{c}94 \\
(0,0078)\end{array}$ & $\begin{array}{c}113 \\
(0,0041)\end{array}$ & $\begin{array}{c}90 \\
(0,0048)\end{array}$ & $\begin{array}{c}187 \\
(0,0031)\end{array}$ & $\begin{array}{c}272 \\
(0,0041)\end{array}$ & $\begin{array}{c}178 \\
(0,0027)\end{array}$ \\
\hline B & 18 & - & - & - & $\begin{array}{c}99 \\
(0,0057)\end{array}$ & $\begin{array}{c}94 \\
(0,0053)\end{array}$ & $\begin{array}{c}151 \\
(0,0103)\end{array}$ & - & - & - \\
\hline \multirow{2}{*}{ C } & 8 & $\begin{array}{c}54 \\
(0,0084)\end{array}$ & $\begin{array}{c}98 \\
(0,0169)\end{array}$ & $\begin{array}{c}42 \\
(0,0139)\end{array}$ & $\begin{array}{c}41 \\
(0,0117)\end{array}$ & $\begin{array}{c}87 \\
(0,0044)\end{array}$ & $\begin{array}{c}40 \\
(0,0042)\end{array}$ & $\begin{array}{c}83 \\
(0,0041)\end{array}$ & $\begin{array}{c}132 \\
(0,0006)\end{array}$ & $\begin{array}{c}73 \\
(0,0032)\end{array}$ \\
\hline & 10 & - & - & - & $\begin{array}{c}135 \\
(0,0038)\end{array}$ & $\begin{array}{c}147 \\
(0,0027)\end{array}$ & $\begin{array}{c}139 \\
(0,0026)\end{array}$ & - & - & - \\
\hline$A, B$ et $C$ & 47 & 66 & 97 & 66 & 96 & 109 & 115 & 143 & 213 & 133 \\
\hline
\end{tabular}

Prélèvement à l'anse calibrée dans la minute qui suit l'agitation (a), après 10 min dans la couche superficielle (b), après 10 min et élimination de la couche superficielle (c).

Entre parenthèses : variance de répétabilité (erreur résiduelle) de la méthode de numération calculée sur les logarithmes de base 10 . 
cette étude que dans la précédente ; elle paraît aussi, meilleure avec le mode d'agitation UT (Ultraturrax) qu'avec les deux autres modes d'agitation ; cela est normal puisque, théoriquement, la variance de répétabilité diminue lorsque le nombre de colonies augmente [11]. Il est intéressant de noter encore que le laboratoire $\mathrm{C}$ qui a mécanisé le prélèvement à l'anse calibrée n'atteint pas une précision supérieure à celle des laboratoires qui utilisent la méthode manuelle confirmant ainsi les résultats de l'un de nous [3].

\section{CONCLUSION}

Le meilleur mode d'agitation des échantillons de lait en vue du dénombrement de la flore totale est, en principe, celui qui disperse le plus complètement les amas microbiens ; on réduit ainsi au minimum une des causes principales d'écarts dans les numérations obtenus par des laboratoires ou par des opérateurs différents. C'est actuellement l'agitation par turbine (Ultraturrax ou appareils équivalents) qui, de ce point de vue, permet d'obtenir les meilleurs résultats [11]. Malheureusement, il est difficile de la mettre en œuvre lorsqu'on doit examiner un grand nombre d'échantillons, comme c'est le cas des laboratoires chargés des analyses d'échantillons, comme c'est le cas des laboratoires chargés des analyses en vue du paiement du lait suivant sa qualité. De plus, ce traitement du lait provoque l'apparition d'une épaisse couche de mousse relativement stable qui gêne considérablement le prélèvement avec l'anse ; il n'est donc pas facilement applicable aux dénombrements en série.

Inversement, une agitation faible consistant, par exemple, en de simples retournement du flacon, conduit à une nette sous-estimation du nombre de micro-organismes présents dans le lait. Cette sousestimation dépend de l'origine de ce dernier (donc de sa composition et/ou de la nature de la flore qu'il contient) comme en témoignent les valeurs élevées de l'interaction "Echantillons x Méthodes d'agitation ».

Le meilleur compromis entre ces deux extrêmes paraît être l'agitation "standard » (mode AS) qui peut être facilement mécanisée. Habituellement, on attend que la mousse formée se dissipe spontanément pour faire le prélèvement, les échantillons étant souvent conservés à température ambiante. Cette pratique peut entraîner, d'un laboratoire à l'autre, des écarts dans les résultats obtenus à partir d'échantillons d'une même origine. Ces divergences constatées dans les deux expériences que nous avons faites, sont plus vraisemblablement liées à une remontée de crème (et de fines bulles d'air) qu'à un multiplication microbienne. C'est ce que suggère le fait qu'après 
élimination de la couche superficielle de lait (deuxième expérience), on obtienne des résultats comparables à ceux de l'analyse réalisée immédiatement après l'agitation. Après leur agitation les échantillons devraient être conservés au froid (moins de $5^{\circ} \mathrm{C}$ ) le temps nécessaire à la disparition de la couche de mousse et agités de nouveau, de façon modérée (par inversion), juste avant l'analyse, afin de disperser la couche superficielle du lait.

Le réchauffement de l'échantillon de lait, destiné à faciliter la dispersion de la couche de crème, peut favoriser la multiplication microbienne si le prélèvement avec l'anse intervient après une attente supérieure à $10 \mathrm{~min}$ [5]. Cela risque de se produire dans le cas d'analyses en séries, du fait d'un arrêt momentané de l'appareil à ensemencer, ou tout simplement du décalage de temps entre le premier et le dernier échantillon analysé. Il est donc déconseillé.

\section{Résumé}

L'influence de divers modes d'agitation du lait sur les résultats de dénombrement de la flore microbienne suivant la méthode Thompson a été étudiée dans trois laboratoires. Deux d'entre eux effectuaient manuellement le prélèvement du lait avec la boucle calibrée, alors que le troisième faisait ces prélèvements à l'aide d'un mécanisme approprié. Des différences significatives entre modes d'agitation ont été mises en évidence, ainsi que l'effet d'une attente entre le moment de l'agitation du lait et celui de son analyse. Sur le plan de la répétabilité des dénombrements, aucune différence significative n'est apparue entre les diverses agitations, ni entre prélèvement manuel et prélèvement mécanisé avec l'anse calibrée.

Compte tenu des résultats acquis et des observations faites au cours de l'étude, l'agitation décrite dans «Standard Method for the Examination of Dairy Product " est recommandée pour le dénombrement suivant la méthode Thompson. Après une attente suffisante à moins de $5^{\circ} \mathrm{C}$ pour éliminer la mousse, l'échantillon doit être à nouveau agité légèrement et analysé immédiatement.

\section{S u m m a r y}

COMPARISON OF DIFFERENT SHAKING METHODS OF RAW MILK SAMPLES BEFORE ENUMERATION BY THE PLATE LOOP METHOD

Using the Plate Loop Count method for enumerating bacteria in refrigerated raw milk, different shaking procedures were compared. Significant differences between them were observed, the more vigou- 
rous method giving from 50 to 100 per cent more bacteria than the conventional one.

The surface of the milk, 10 minutes or more after a vigourous shaking, contains more bacteria than the beneath layer.

Considering the repetability of the Plate Loop method, there is no significant differences between the shaking methods.

According to the results obtained, the shaking procedure recommanded in "Standard Methods for the Examination of Dairy Products" is recommanded with sufficient delay at less than $5^{\circ} \mathrm{C}$ for eliminating the foam and gentle agitation of the milk just before analysis.

Reçu pour publication en octobre 1980.

\section{Bibliographie}

[1] Anonyme (1967). - Studiegroep kwaliteisbepaling melk van de F.N.Z. De bepaling van het kiemgetal van melk et behulp van een öse. 763-766.

[2] Anonyme (1972). - Standard Methods for the examination of dairy products. American Public Health Association. 13th ed., New York.

[3] Grappin (R.) (1975). - Mise au point sur les appareils automatiques utilisés pour la numération des germes totaux du lait ; préparation des boîtes de Petri et comptage des colonies. Rev. Lait. Franç., 335, 629-641.

[4] Huhtanen (C. N.), Brazis (A. R.), Arledge (W. L.), Cook (E. W.), Donnelly (C. B.), Ginn (R. E.), Murphy (J. N.), Randolph (H. E.), Sing (E. L.) and THompson (D. I.) (1970). - Effect of dilution bottle mixing methods on plate counts of raw milk bacteria. J. Milk Food Technol., 33, 269-273.

[5] Huhtanen (C. N.), Brazis (A. R.), Arledge (W. L.), CooK (E. W.) Donnelly (C. B.), GinN (R. E.), Jezeski (J. J.), Pusch (D.), Randolph (H. E.) and SING (E. L.) (1972). - Effects of time of holding dilutions on counts of bacteria from raw milk. J. Milk Food Technol., 35, 126-130.

[6] LeEsment (H.) (1964). - Undersökning av tva enkla plattspridningsmetoder för bestämning av bakterieantalet i kyllagrad leverantörmjölk. Svenska Mejeritidn., 56, 143-149.

[7] Leesment (H.) und Nickels (C. H.) (1966). - Das Eichen der Burri-öse für die Bestimmung der Keimzahl nach dem Plattenverfahren. XVII Congr. Int. Lait, Munich, 481-483.

[8] OrR (M. J.), Baines (S.) and Mc Crae (G. M. C.) (1971). - The plate loop method for determining bacterial numbers in bulk cooled milk. Milk Ind., 68, 17-22.

[9] Posthumus (G.), Klijn (C. J.) and Giesen (Th. J. J.) (1974), - A mechanized loop method for total count of bacteria in refrigerated suppliers' milk. Neth. Milk Dairy J., 28, 79-92.

[10] Richard (J.) et Auclair (J.) (1968). - Méthode simplifiée de dénombrement des germes totaux du lait. Rev. Lait. Franç., 261, 15-18.

[11] Richard (J.) (1980). - Influence de l'agitation du lait cru sur les résultats de dénombrement de sa flore totale à l'aide d'une anse calibrée. Le Lait, $L X, 211-225$. 
[12] SNedecor (G. W.) and Cochran (W. G.) (1967). - Statistical methods. The Iowa State University Press, $6^{\mathrm{e}}$ ed., Ames Iowa (U.S.A.).

[13] Tatini (S. R.), Dabbah (R.) and Olson Jr. (J. C.) (1967). - Comparison of plate loop and agar plate methods for bacteriological examination of manufacturing grade raw milk. J. Milk Food Technol., 30, 112-115.

[14] Thompson (D. I.), Donnelly (C. B.) and Black (L. A.) (1960). - A plate loop method for determining viable counts of raw milk. J. Milk Food Technol., 23, 167-171.

[15] Wanser (B. E.) and Hartman (P. A.) (1962). - Agitation of milk samples for colony counts of raw milk. J. Milk Food Technol., 25, 5-6.

[16] Wright (E. O.), Reinbold (G. W.), Burmeister (L.) and Mellon (J.) (1970). Prediction of standard plate count of manufacturing-grade raw milk from the plate loop count. J. Milk Food Technol., 33, 168-170. 